\title{
Which Wave Numbers Determine the Thermodynamic Stability of Soft Matter Quasicrystals?
}

\author{
D. J. Ratliff,,$^{1, *}$ A. J. Archer $\oplus^{1, \dagger}$ P. Subramanian $\odot,{ }^{2,3, \ddagger}$ and A. M. Rucklidge $\odot^{2, \S}$ \\ ${ }^{1}$ Department of Mathematical Sciences and Interdisciplinary Centre for Mathematical Modelling, \\ Loughborough University, Loughborough, Leicestershire LE11 3TU, United Kingdom \\ ${ }^{2}$ School of Mathematics, University of Leeds, Leeds LS2 9JT, United Kingdom \\ ${ }^{3}$ Mathematical Institute, University of Oxford, Oxford OX2 6GG, United Kingdom
}

(Received 15 July 2019; published 1 October 2019)

\begin{abstract}
For soft matter to form quasicrystals an important ingredient is to have two characteristic length scales in the interparticle interactions. To be more precise, for stable quasicrystals, periodic modulations of the local density distribution with two particular wave numbers should be favored, and the ratio of these wave numbers should be close to certain special values. So, for simple models, the answer to the title question is that only these two ingredients are needed. However, for more realistic models, where in principle all wave numbers can be involved, other wave numbers are also important, specifically those of the second and higher reciprocal lattice vectors. We identify features in the particle pair interaction potentials that can suppress or encourage density modes with wave numbers associated with one of the regular crystalline orderings that compete with quasicrystals, enabling either the enhancement or suppression of quasicrystals in a generic class of systems.
\end{abstract}

DOI: 10.1103/PhysRevLett.123.148004

Matter does not normally self-organize into quasicrystals (QCs). Regular crystalline packings are much more common in nature and some specific ingredients are required for $\mathrm{QC}$ formation, which is why the first QCs were not identified until 1982, in certain metallic alloys [1]. Subsequently, the seminal work in Refs. [2,3] showed that normally a crucial element in QC formation, at least in soft matter, is the presence of two prominent wave numbers in the linear response behavior to periodic modulations of the particle density distribution. This is equivalent to having two prominent peaks in the static structure factor or in the dispersion relation $[4,5]$. In soft matter systems, the effective interactions between molecules and aggregations of molecules (generically referred to here as particles) can be tuned to exhibit the two specific required length scales and thus form QCs. Such systems include block copolymers and dendrimers [6-15], certain anisotropic particles [16-18], nanoparticles [19,20] and mesoporous silica [21].

Some of our understanding of how and why QCs can form has come from studies of particle-based computer simulation models-see, for example, Refs. [22-26]. Another source of important insights has been continuum theories for the density distribution. The earliest of these consist of generalized Landau-type order-parameter theories [2,3,27-34]. More recently, classical density functional theory (DFT) [35-37] in conjunction with its dynamical extension DDFT [38-40] has been utilized. DFT is a statistical mechanical theory for the distribution of the average particle number density that takes as input the particle pair interaction potentials, and so bridges between particle-based and Landau-type continuum theory approaches. The DFT results for QC forming systems [4,5,41-43] clearly demonstrate how the crucial pair of prominent wave numbers are connected to the length and energy scales present in the pair potentials.

While the ratio between the two length scales is important, it can be seen that this is not the whole story if one compares the phase behavior of systems with the pair potential of Ref. [42] (phase diagrams are calculated below) with the phase behavior of the core-shoulder soft potential system of Refs. [4,5]. We refer to these two as the BEL and ARK models, respectively. In the ARK model, QCs are never the thermodynamic equilibrium phase, i.e., the state which is the global minimum of the free energy, and they only form in this system for subtle dynamical reasons $[4,5]$. In contrast, QCs can be the thermodynamic equilibrium for the BEL model. This is despite the fact that the parameters in both the BEL and ARK models are chosen so that both systems have identical growth rates $\omega$ at the two critical wave numbers $k_{1}$ and $k_{q}$, so that density fluctuations with these two wave numbers are promoted equally in the two different systems. This raises the important question: what feature(s) do BEL-type systems have that enables QCs to be thermodynamically stable, that ARK-type systems do not have? Or, relating to the title question, why is it not enough to consider just these two wave numbers?

The answer to this question is that one must also consider the properties of the dispersion relation $\omega(k)$ at certain other wave numbers $\neq k_{1}, k_{q}$ For example, in two dimensions (2D), hexagonal crystals are built up from six modes 
$\sim \exp (i \mathbf{k} \cdot \mathbf{r})$ at $60^{\circ}$ to one another with equal (single) wave number $k=|\mathbf{k}|$. They are stabilized by nonlinear coupling between these modes and modes with wave numbers such as $\sqrt{3} k, 2 k$, and $\sqrt{7} k$, which are generated by vector sums of the original six. The resulting wave vectors are the hexagonal reciprocal lattice vectors (RLVs). More generally, with two wave numbers, more complex structures can form and involve larger sets of RLVs. The properties of modes with these vectors, in particular their decay rates $\omega$, must be known in order to predict which structures have the lowest free energy.

We illustrate this fundamental understanding by developing a class of model systems with pair potentials which have identical growth rates at $k_{1}$ and $k_{q}$, but are different in a controllable manner at the RLV wave numbers. By changing the dispersion relation at these wave numbers, we are able either to enhance or suppress the stability of QCs.

While it is not a priori obvious that soft matter freezing might be related to Faraday waves, it turns out that a surprisingly large amount of the mathematics of Faraday wave pattern formation can be applied to the soft matter systems of interest here, including the understanding of QC stability $[2,44-51]$. Faraday waves are standing waves on the surface of viscous liquid layers that arise when the liquid is subjected to strong enough vertical vibrations [52]. In some circumstances, Faraday wave experiments exhibit spatially complex patterns such as 12-fold quasipatterns at parameters where two length scales in the correct ratio are excited or weakly damped [44,51,53-58]. A major conclusion from this body of work is that understanding spatially complex patterns in Faraday waves requires the consideration of not only the primary waves in the pattern but also the contributions from the RLV waves. These RLV contributions are strongly influenced by the damping rate at each wave number. We demonstrate here that analogous mechanisms operate in the coupling between soft matter density modulations at different wave numbers, helping to identify features in the pair potentials that can be tuned to control the extent to which the QCs are stabilized.

For a system of interacting particles free of any external forces, the equilibrium density distribution $\rho(\mathbf{r})$ is given by the minimum of the grand potential functional [35-37]

$\Omega[\rho]=k_{B} T \int \rho\left(\ln \left(\Lambda^{d} \rho\right)-1\right) d \mathbf{r}+\mathcal{F}_{\text {ex }}[\rho]-\mu \int \rho d \mathbf{r}$,

where $\Lambda$ is the thermal de Broglie wavelength, $k_{B}$ is Boltzmann's constant, $T$ is the temperature, and $\mu$ is the chemical potential. In two dimensions, we have $d=2$ and $\mathbf{r}=(x, y)$. We illustrate the main ideas of this Letter in two dimensions, but they equally apply in three dimensions. The first term in Eq. (1) is the entropic ideal-gas contribution to the Helmholtz free energy, while the second term is the excess contribution, which arises from the interactions between particles. The random phase approximation (RPA) [35,59]

$$
\mathcal{F}_{\text {ex }}[\rho]=\frac{1}{2} \iint \rho(\mathbf{r}) V\left(\left|\mathbf{r}-\mathbf{r}^{\prime}\right|\right) \rho\left(\mathbf{r}^{\prime}\right) d \mathbf{r} d \mathbf{r}^{\prime},
$$

turns out to be remarkably accurate for soft particles interacting pairwise via potentials $V(r)$, which are finite for all values of the separation distance $r$ between the particles [59] and so is used here. Equilibrium density profiles minimize Eq. (1) and so satisfy the Euler-Lagrange equation $(\delta \Omega / \delta \rho)=0$. In the liquid state, the density is uniform, while in the crystal and QC phases the profiles are nonuniform, typically with sharp peaks.

An understanding of how the thermodynamic equilibrium structures are selected comes from rewriting Eq. (2) in Fourier space:

$$
\mathcal{F}_{\mathrm{ex}}=\frac{1}{2(2 \pi)^{d}} \int \hat{V}(k)|\hat{\rho}(\mathbf{k})|^{2} d \mathbf{k},
$$

where $\hat{\rho}(\mathbf{k})=\int e^{-i \mathbf{k} \cdot \mathbf{r}} \rho(\mathbf{r}) d \mathbf{r}$ is the Fourier transform of the density profile $\rho(\mathbf{r})$ and $\hat{V}(k)$ is similarly defined as the Fourier transform of $V(r)$. We observe that density modes $\hat{\rho}(\mathbf{k})$ with wave numbers at the minima of $\hat{V}(k)$ minimize the above integral, whereas those with wave numbers away from these values make a larger contribution to $\mathcal{F}_{\text {ex }}$ and so are favored less. In other words, $\hat{V}(k)$ quantifies the energetic penalty for having modes with wave number $k$ in the density profile. Of course, the entropic ideal-gas term in Eq. (1) also makes an important contribution. This is particularly true near to melting, which is where the soft QCs discussed here exist.

Assuming that the particles have overdamped Brownian equations of motion, the nonequilibrium dynamics of the density distribution $\rho(\mathbf{r}, t)$ is given by DDFT [38-40]

$$
\frac{\partial \rho}{\partial t}=\Gamma \nabla \cdot\left(\rho \nabla \frac{\delta \Omega}{\delta \rho}\right)
$$

where $t$ is time and $\Gamma$ is a mobility coefficient. The stability of a uniform liquid state of density $\rho_{0}$ to small amplitude perturbations $\sim \exp (i \mathbf{k} \cdot \mathbf{r}+\omega t)$ can be found by a standard normal mode approach $[4,5,39,60]$, which gives the linear dispersion relation for the growth (or decay) rate $\omega$ associated with modes of wave number $k$,

$$
\omega(k)=-D k^{2}\left[1+\rho_{0} \beta \hat{V}(k)\right],
$$

where $D=\Gamma k_{B} T$ is the diffusion coefficient and $\beta=$ $\left(k_{B} T\right)^{-1}$. In Eq. (5) the first term $\left(-D k^{2}\right)$ stems from the ideal-gas contribution and is entropic in origin, while the second term $\left[-D \rho_{0} \beta k^{2} \hat{V}(k)\right]$ is the energetic contribution. The liquid is dynamically stable when $\omega(k)<0$ for all $k>0$, but becomes unstable at critical wave number(s) 
TABLE I. The three sets of parameter values used in the BEL pair potential (6). With these values and $\rho_{0}=1.25$ the systems are simultaneously marginally stable to modes with $k_{1}$ and $k_{q}$.

\begin{tabular}{lllcll}
\hline \hline$\sigma$ & \multicolumn{1}{c}{$C_{0}$} & \multicolumn{1}{c}{$C_{2}$} & \multicolumn{1}{c}{$C_{4}$} & \multicolumn{1}{c}{$C_{6}$} & \multicolumn{1}{c}{$C_{8}$} \\
\hline 0.794 & 1.350 & 1.794 & 0.7224 & 0.08368 & 0.003117 \\
0.771 & 1.000 & 1.095 & 0.4397 & 0.04927 & 0.001831 \\
0.671 & 0.3949 & 0.04485 & 0.03689 & 0.003342 & 0.0001449 \\
\hline \hline
\end{tabular}

$k=k_{c}$ if $\omega\left(k_{c}\right)=0$ at a local maximum. This can only happen if $\hat{V}(k)<0$ for some range of $k$ [61], and then the instability occurs through increasing $\rho_{0}$ or decreasing $T$. For the class of two length scale systems here, there are two maxima in $\omega(k)$, at $k_{1}$ and $k_{q}$. The ratio between these is important for determining the structures formed, but as we now show, other wave numbers in the reciprocal lattice are important too.

We demonstrate this by modifying a pair potential $V(r)$ in such a way that $\hat{V}(k)$ remains fixed at $k_{1}$ and $k_{q}$ but changes everywhere else, strongly affecting which structures minimize Eq. (1) and so are the thermodynamic equilibria. We use the form of the BEL pair potential [42]:

$V(r)=\varepsilon e^{-\frac{1}{2} \sigma^{2} r^{2}}\left(C_{0}+C_{2} r^{2}+C_{4} r^{4}+C_{6} r^{6}+C_{8} r^{8}\right)$.

Throughout we set $\beta \epsilon=10$ and the remaining parameters $\left\{\sigma, C_{0}, C_{2}, C_{4}, C_{6}, C_{8}\right\}$ have values chosen so that the dispersion relation has two maxima at $k_{1}=1$ and $k_{q}=$ $q=2 \cos (\pi / 12) \approx 1.93$ [minima in $\hat{V}(k)$ ], but varies significantly for other $k$ values. We choose three sets of parameter values, given in Table I, in order to enhance or reduce the energetic cost at other RLV wave numbers. The middle set, with $\sigma=0.771$, are the values originally used in Ref. [42] and the other two sets are for a larger and smaller value of $\sigma$. The resulting dispersion relations, Fourier transforms of the pair potentials $\hat{V}(k)$ and the potentials $V(r)$ in real space are displayed in Fig. 1.

From Fig. 1(b), we see that decreasing $\sigma$ results in $\hat{V}(k)$ being more damped at larger $k$ values and thus leads to a lower energetic penalty [see Eq. (3)] at the hexagonal RLV wave numbers of $k_{1}$ and $k_{q}$, i.e., at the wave numbers $\sqrt{3} k_{1}$, $2 k_{1}, \sqrt{3} k_{q}$, and $2 k_{q}$, which are marked as vertical gray lines in Figs. 1(a) and 1(b). In contrast, increasing $\sigma$ leads to a higher penalty at the hexagonal RLV wave numbers. There are corresponding changes to the decay rates $\omega(k)$ [Fig. 1(a)]. The important QC RLV wave numbers are $k_{1} / q, \sqrt{2} k_{1}, \sqrt{2} k_{q}$, and $q k_{q}$ (dashed gray lines), and there are of course also changes in the value of $\omega$ at these wave numbers as $\sigma$ is varied. However, on decreasing $\sigma$ the biggest fractional change in $\omega$ occurs at wave number $\sqrt{3} k_{q}$, where $|\omega|$ decreases by $90 \%$ going from $\sigma=0.794$ to $\sigma=0.671$, while the change at $\sqrt{2} k_{q}$ is $88 \%$ and at all
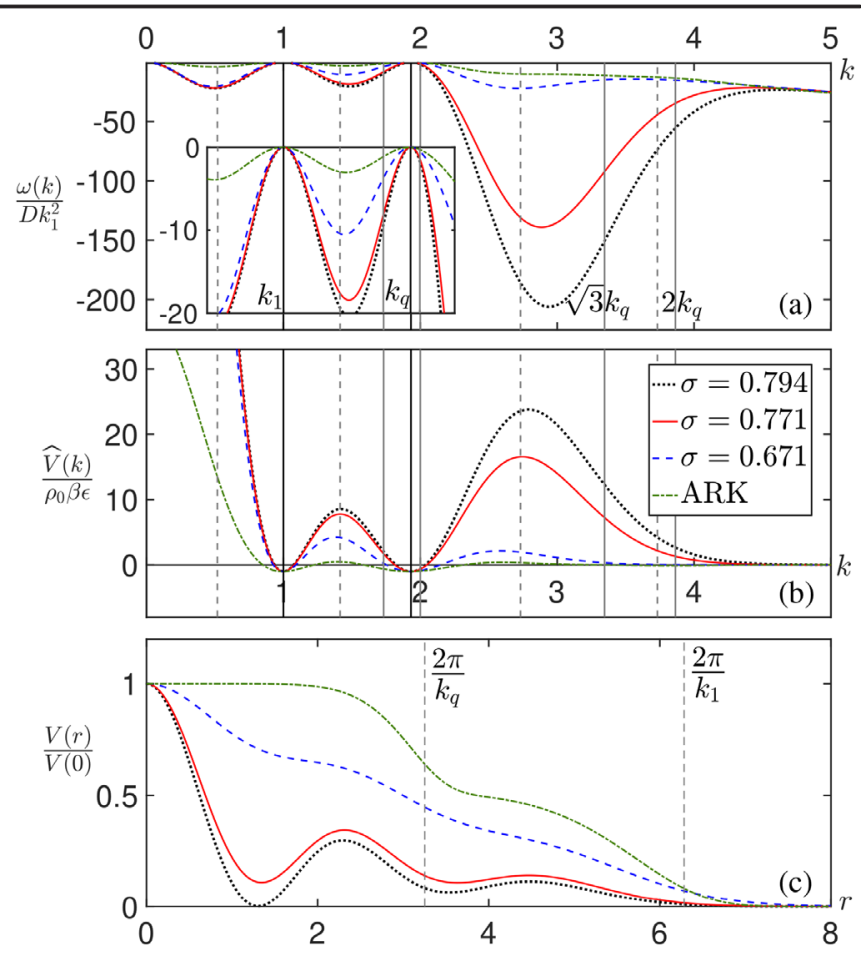

FIG. 1. In (a) the dispersion relation $\omega(k)$ (5) for three different BEL and the ARK potentials (the inset shows a magnification) at the state point where they are simultaneously marginally stable at $k_{1}$ and $k_{q}$, which are marked with vertical black lines. Some other important RLV wave numbers for hexagons are marked as vertical gray lines and for QCs as dashed gray lines. In (b) we display the corresponding pair potentials in Fourier space and in (c) the pair potentials in real space with the two key length scales marked. The $\sigma$ values for the three BEL systems are given in the key and the remaining parameters are given in Table I.

other key wave numbers the fractional change is significantly smaller. Therefore, hexagons with wave number $k_{q}$ ( $q$-hex) should be stabilized more than QCs by the decrease in $\sigma$, which we confirm below by calculating free energies and phase diagrams - see Figs. 2 and 3.

We also display in Fig. 1 the ARK model pair potential $V(r)=\epsilon\left(e^{-\left(r / R_{c}\right)^{8}}+a e^{-\left(r / R_{s}\right)^{8}}\right)$ and corresponding $\hat{V}(k)$ and $\omega(k)$. We choose the parameter values $\left\{\epsilon, a, R_{c}, R_{s}\right\}$ so that the system is identical to that studied in Refs. [4,5], i.e., with $R_{s}=1.855 R_{c}$ and $a=1.067$, where the phase diagram was also determined. Here we rescale the core and shoulder radii $R_{c}$ and $R_{s}$ by choosing $R_{c}=3.14$, so that the critical wave numbers are at $k_{1}=1$ and $k_{q}=q$ as in the three chosen BEL potentials (6). This rescaling does not in any way change the phase behavior.

Figure 1(c) illustrates how varying the parameters changes the architecture of the potentials in physical space. Increasing $\sigma$ (together with changes to the other parameters) leads to oscillations in the BEL potential becoming accentuated, to the extent that the first minimum at $r \approx 1.3$ comes close to zero in the $\sigma=0.794$ case. On the other hand, the opposite changes smooth the oscillations, to the 


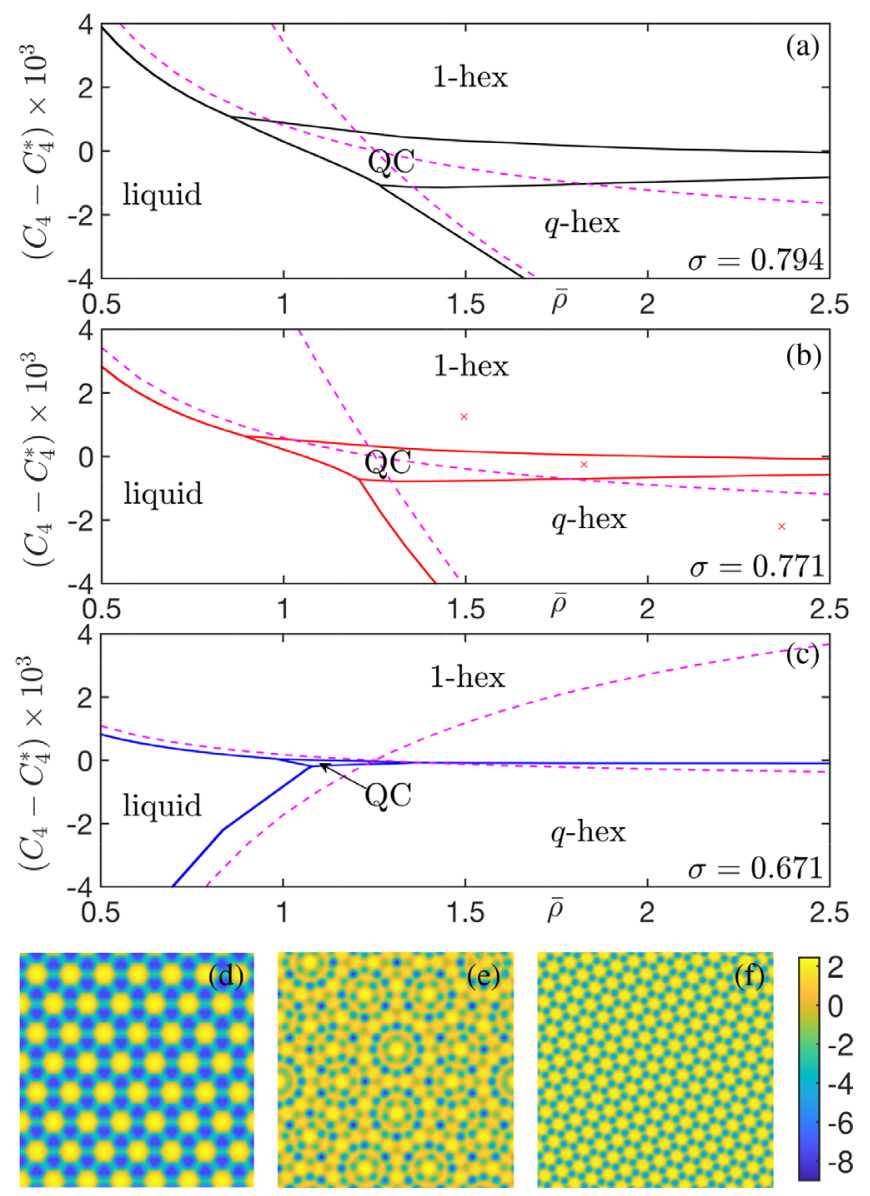

FIG. 2. Phase diagrams for the BEL model in the average density $\bar{\rho}$ versus the $C_{4}-C_{4}^{*}$ plane. The critical value of $C_{4}=C_{4}^{*}$ for three different values of $\sigma$ is given in Table I, as are the other pair potential parameter values, which remain fixed. There are four equilibrium phases: a uniform liquid phase, a large lattice spacing hexagonal phase (1-hex), a smaller lattice spacing hexagonal phase $(q$-hex) and QCs. For $\sigma=0.771$, examples of the observed nonuniform phases are displayed in (d)-(f). We plot $\ln \rho(\mathbf{r})$; the color bar indicates the range of values. These three are calculated at the state points marked with a $\times$ symbol in the phase diagram (b). The coexistence regions between the different phases are rather narrow and within the widths of the lines used. We also display the liquid linear stability threshold lines (dashed lines). There are two, one corresponding to instability at $k_{1}$ and the other at $k_{q}$. They intersect at the point where both length scales are marginally stable, which occurs at $\bar{\rho}=\rho_{0}=1.25$ in all three systems.

point where it becomes hard, in real space, to discern more than one length scale. The BEL potential with $\sigma=0.671$ bears some resemblance to the ARK potential.

In Fig. 2 we display equilibrium phase diagrams, computed by varying $\beta \mu$ and $C_{4}$, and minimizing the grand potential (1) via Picard iteration [4,62]. Varying just $C_{4}$ changes the BEL potential in a manner most akin to varying $a$ in the ARK potential [43]. The top three panels are for the three chosen BEL potentials, and show the equilibrium phase as a function of average density $\bar{\rho}$ and

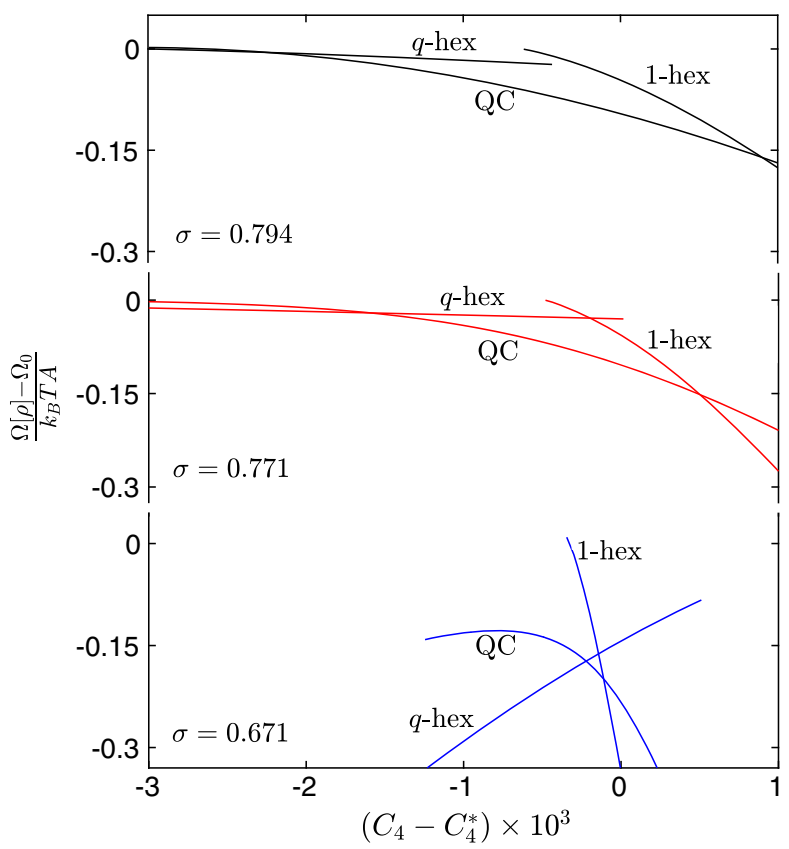

FIG. 3. Grand potential for the 1-hex, $q$-hex, and QCs for varying $C_{4}$, for $\beta \mu=224$ for the three different BEL systems. The corresponding $\sigma$ values are indicated.

$C_{4}-C_{4}^{*}$. Here, $C_{4}^{*}$ is the value of $C_{4}$ for simultaneous marginal stability, as given in Table I. Varying $C_{4}$ away from $C_{4}^{*}$ means that the maxima in $\omega(k)$ are no longer at the same value, shifting the preference to one or the other length scale [43]. Typical examples of the density profiles obtained are displayed along the bottom of Fig. 2.

Figure 3 shows examples of the grand potential per unit area $A$ (relative to the value for the liquid $\Omega_{0} / A$ ) as a function of $C_{4}$ at constant $\beta \mu=224$. In these plots, the thermodynamic equilibrium phase is that with the lowest value of $\Omega$ for the given value of $C_{4}$. The crossing points of the different branches in each case give the phase boundaries displayed in Fig. 2.

The size of the region where QCs are stable in each phase diagram in Fig. 2 comes from the changes to the potentials shown in Fig. 1. Case (a) with the larger $\sigma=0.794$ has QCs as the thermodynamic equilibrium over a much larger region of the phase diagram than (c), with the smaller $\sigma=0.671$, where they are almost completely suppressed. In the ARK phase diagram displayed in Refs. [4,5], QCs are completely absent. The reason for these significant changes is that decreasing $\sigma$ and thus making $\omega(k)$ less negative away from $k_{1}$ and $k_{q}$ [see Fig. 1(a)] benefits all phases that incorporate other wave numbers, but benefits most the $q$-hex crystals, as discussed above. In common with Faraday waves, wave numbers that are less strongly damped play a more prominent role in selecting the final state [63]. Of course, determining the thermodynamic equilibrium involves a nonlinear balance between contributions from all RLV wave numbers, but our results in 
Figs. 2 and 3 are consistent with this intuition from Faraday waves. That one can accurately anticipate the (nonlinear) phase behavior from the linear response behavior $\omega(k)$ was not a priori obvious and is an important result. But, this may not be generally true if the approximation for $\mathcal{F}_{\text {ex }}$ used is more complex than in Eq. (2).

A simplification that is made in some other models is to introduce a coefficient (the parameter $c$ in Refs. [28,29,34,64] or $\gamma$ in Ref. [32]) which effectively sends $\omega(k) \rightarrow-\infty$ for all wave numbers $k \neq k_{1}, k_{q}$ as $c \rightarrow \infty$. This limit of perfect length scale selectivity makes the resulting pair interaction potentials less physically realizable. The present approach does not rely on this simplification and is therefore more relevant to elucidating QC formation in soft matter at finite temperatures.

To conclude, we return to the title question: As Refs. [2,3] showed and subsequent work confirmed, two wave numbers $k_{1}$ and $k_{q}$ having a specific ratio are required for quasicrystals to be stable, i.e., a local minimum of the grand potential. However, what we have shown here is that for QCs to be the thermodynamic equilibrium, one must also consider the RLV wave numbers of all competing crystal structures. Moreover, examining the value of the dispersion relation $\omega(k)$ at these other RLV wave numbers helps anticipate the outcome of the competition between QCs and other crystal structures.

This work was supported in part by a L'Oréal UK and Ireland Fellowship for Women in Science (P. S.), by the EPSRC under Grants EP/P015689/1 (A. J. A., D. J. R.) and EP/P015611/1 (A.M.R.), and by the Leverhulme Trust (RF-2018-449/9, A. M. R.). We would like to thank Ron Lifshitz, Sam Savitz, and Ken Elder for various helpful discussions during the formulation of this Letter.

*D.J.Ratliff@lboro.ac.uk

†A.J.Archer@lboro.ac.uk

*Priya.Subramanian@maths.ox.ac.uk

§A.M.Rucklidge@leeds.ac.uk

[1] D. Shechtman, I. Blech, D. Gratias, and J. W. Cahn, Metallic Phase with Long-Range Orientational Order and No Translational Symmetry, Phys. Rev. Lett. 53, 1951 (1984).

[2] R. Lifshitz and D. M. Petrich, Theoretical Model for Faraday Waves with Multiple-Frequency Forcing, Phys. Rev. Lett. 79, 1261 (1997).

[3] R. Lifshitz and H. Diamant, Soft quasicrystals-Why are they stable? Philos. Mag. 87, 3021 (2007).

[4] A. J. Archer, A. M. Rucklidge, and E. Knobloch, Quasicrystalline Order and a Crystal-Liquid State in a Soft-Core Fluid, Phys. Rev. Lett. 111, 165501 (2013).

[5] A. J. Archer, A. M. Rucklidge, and E. Knobloch, Soft-core particles freezing to form a quasicrystal and a crystal-liquid phase, Phys. Rev. E 92, 012324 (2015).

[6] X. Zeng, G. Ungar, Y. Liu, V. Percec, A. E. Dulcey, and J. K. Hobbs, Supramolecular dendritic liquid quasicrystals, Nature (London) 428, 157 (2004).
[7] K. Hayashida, T. Dotera, A. Takano, and Y. Matsushita, Polymeric Quasicrystal: Mesoscopic Quasicrystalline Tiling in ABC Star Polymers, Phys. Rev. Lett. 98, 195502 (2007).

[8] S. Fischer, A. Exner, K. Zielske, J. Perlich, S. Deloudi, W. Steurer, P. Lindner, and S. Förster, Colloidal quasicrystals with 12-fold and 18-fold diffraction symmetry, Proc. Natl. Acad. Sci. U.S.A. 108, 1810 (2011).

[9] S. C. Glotzer and M. Engel, Complex order in soft matter, Nature (London) 471, 309 (2011).

[10] C. R. Iacovella, A. S. Keys, and S. C. Glotzer, Self-assembly of soft-matter quasicrystals and their approximants, Proc. Natl. Acad. Sci. U.S.A. 108, 20935 (2011).

[11] J. Zhang and F.S. Bates, Dodecagonal quasicrystalline morphology in a poly (styrene-b-isoprene-b-styrene-bethylene oxide) tetrablock terpolymer, J. Am. Chem. Soc. 134, 7636 (2012).

[12] S. Lee, C. Leighton, and F. S. Bates, Sphericity and symmetry breaking in the formation of Frank-Kasper phases from one component materials, Proc. Natl. Acad. Sci. U.S.A. 111, 17723 (2014).

[13] T. M. Gillard, S. Lee, and F. S. Bates, Dodecagonal quasicrystalline order in a diblock copolymer melt, Proc. Natl. Acad. Sci. U.S.A. 113, 5167 (2016).

[14] K. Yue, M. Huang, R. L. Marson, J. He, J. Huang, Z. Zhou, J. Wang, C. Liu, X. Yan, K. Wu et al., Geometry induced sequence of nanoscale Frank-Kasper and quasicrystal mesophases in giant surfactants, Proc. Natl. Acad. Sci. U.S.A. 113, 14195 (2016).

[15] M. Huang, K. Yue, J. Wang, C.-H. Hsu, L. Wang, and S. Z. D. Cheng, Frank-Kasper and related quasicrystal spherical phases in macromolecules, Sci. China: Chem. 61, 33 (2018).

[16] A. Haji-Akbari, M. Engel, A. S. Keys, X. Zheng, R. G. Petschek, P. Palffy-Muhoray, and S. C. Glotzer, Disordered, quasicrystalline and crystalline phases of densely packed tetrahedra, Nature (London) 462, 773 (2009).

[17] A. Haji-Akbari, M. Engel, and S. C. Glotzer, Degenerate Quasicrystal of Hard Triangular Bipyramids, Phys. Rev. Lett. 107, 215702 (2011).

[18] J. Dontabhaktuni, M. Ravnik, and S. Žumer, Quasicrystalline tilings with nematic colloidal platelets, Proc. Natl. Acad. Sci. U.S.A. 111, 2464 (2014).

[19] D. V. Talapin, E. V. Shevchenko, M. I. Bodnarchuk, X. Ye, J. Chen, and C.B. Murray, Quasicrystalline order in self-assembled binary nanoparticle superlattices, Nature (London) 461, 964 (2009).

[20] X. Ye, J. Chen, M. E. Irrgang, M. Engel, A. Dong, S. C. Glotzer, and C.B. Murray, Quasicrystalline nanocrystal superlattice with partial matching rules, Nat. Mater. 16, 214 (2017).

[21] C. Xiao, N. Fujita, K. Miyasaka, Y. Sakamoto, and O. Terasaki, Dodecagonal tiling in mesoporous silica, Nature (London) 487, 349 (2012).

[22] M. Engel and H.-R. Trebin, Self-Assembly of Monatomic Complex Crystals and Quasicrystals with a Double-Well Interaction Potential, Phys. Rev. Lett. 98, 225505 (2007).

[23] T. Dotera, T. Oshiro, and P. Ziherl, Mosaic two-lengthscale quasicrystals, Nature (London) 506, 208 (2014).

[24] M. Engel, P. F. Damasceno, C. L. Phillips, and S. C. Glotzer, Computational self-assembly of a one-component icosahedral quasicrystal, Nat. Mater. 14, 109 (2015). 
[25] M. Martinsons and M. Schmiedeberg, Growth of two-dimensional decagonal colloidal quasicrystals, J. Phys. Condens. Matter 30, 255403 (2018).

[26] A. Gemeinhardt, M. Martinsons, and M. Schmiedeberg, Stabilizing quasicrystals composed of patchy colloids by narrowing the patch width, Europhys. Lett. 126, 38001 (2019).

[27] C. V. Achim, M. Schmiedeberg, and H. Löwen, Growth Modes of Quasicrystals, Phys. Rev. Lett. 112, 255501 (2014).

[28] K. Jiang and P. Zhang, Numerical methods for quasicrystals, J. Comput. Phys. 256, 428 (2014).

[29] K. Jiang, J. Tong, P. Zhang, and A.-C. Shi, Stability of twodimensional soft quasicrystals in systems with two length scales, Phys. Rev. E 92, 042159 (2015).

[30] P. Subramanian, A. J. Archer, E. Knobloch, and A. M. Rucklidge, Three-Dimensional Icosahedral Phase Field Quasicrystal, Phys. Rev. Lett. 117, 075501 (2016).

[31] M. Schmiedeberg, C. V. Achim, J. Hielscher, S. C. Kapfer, and H. Löwen, Dislocation-free growth of quasicrystals from two seeds due to additional phasonic degrees of freedom, Phys. Rev. E 96, 012602 (2017).

[32] K. Jiang, P. Zhang, and A.-C. Shi, Stability of icosahedral quasicrystals in a simple model with two-length scales, J. Phys. Condens. Matter 29, 124003 (2017).

[33] P. Subramanian, A. J. Archer, E. Knobloch, and A. M. Rucklidge, Spatially localized quasicrystalline structures, New J. Phys. 20, 122002 (2018).

[34] S. Savitz, M. Babadi, and R. Lifshitz, Multiple-scale structures: From Faraday waves to soft-matter quasicrystals, IUCrJ 5, 247 (2018).

[35] J.-P. Hansen and I. R. McDonald, Theory of Simple Liquids (Elsevier, Oxford, 2013).

[36] R. Evans, The nature of the liquid-vapour interface and other topics in the statistical mechanics of non-uniform, classical fluids, Adv. Phys. 28, 143 (1979).

[37] R. Evans, in Fundamentals of Inhomogeneous Fluids, edited by D. Henderson (Dekker, New York, 1992), Chap. 3.

[38] U. M. B. Marconi and P. Tarazona, Dynamic density functional theory of fluids, J. Chem. Phys. 110, 8032 (1999).

[39] A. J. Archer and R. Evans, Dynamical density functional theory and its application to spinodal decomposition, J. Chem. Phys. 121, 4246 (2004).

[40] A. J. Archer and M. Rauscher, Dynamical density functional theory for interacting Brownian particles: Stochastic or deterministic?, J. Phys. A 37, 9325 (2004).

[41] K. Barkan, H. Diamant, and R. Lifshitz, Stability of quasicrystals composed of soft isotropic particles, Phys. Rev. B 83, 172201 (2011).

[42] K. Barkan, M. Engel, and R. Lifshitz, Controlled SelfAssembly of Periodic and Aperiodic Cluster Crystals, Phys. Rev. Lett. 113, 098304 (2014).

[43] M. C. Walters, P. Subramanian, A. J. Archer, and R. Evans, Structural crossover in a model fluid exhibiting two length scales: Repercussions for quasicrystal formation, Phys. Rev. E 98, 012606 (2018).

[44] W. S. Edwards and S. Fauve, Patterns and quasi-patterns in the Faraday experiment, J. Fluid Mech. 278, 123 (1994).
[45] W. B. Zhang and J. Viñals, Pattern formation in weakly damped parametric surface waves, J. Fluid Mech. 336, 301 (1997).

[46] M. Silber, C. M. Topaz, and A. C. Skeldon, Twofrequency forced Faraday waves: Weakly damped modes and pattern selection, Physica (Amsterdam) 143D, 205 (2000).

[47] J. Porter, C. M. Topaz, and M. Silber, Pattern Control Via Multifrequency Parametric Forcing, Phys. Rev. Lett. 93, 034502 (2004).

[48] J. Porter and M. Silber, Resonant triad dynamics in weakly damped Faraday waves with two-frequency forcing, Physica (Amsterdam) 190D, 93 (2004).

[49] A. M. Rucklidge and M. Silber, Design of parametrically forced patterns and quasipatterns, SIAM J. Appl. Dyn. Syst. 8, 298 (2009).

[50] A.C. Skeldon and G. Guidoboni, Pattern selection for Faraday waves in an incompressible viscous fluid, SIAM J. Appl. Math. 67, 1064 (2007).

[51] A. C. Skeldon and A. M. Rucklidge, Can weakly nonlinear theory explain Faraday wave patterns near onset?, J. Fluid Mech. 777, 604 (2015).

[52] J. Miles and D. Henderson, Parametrically forced surface waves, Annu. Rev. Fluid Mech. 22, 143 (1990).

[53] J. P. Gollub, Order and disorder in fluid motion, Proc. Natl. Acad. Sci. U.S.A. 92, 6705 (1995).

[54] T. Besson, W. S. Edwards, and L.S. Tuckerman, Twofrequency parametric excitation of surface waves, Phys. Rev. E 54, 507 (1996).

[55] H. Arbell and J. Fineberg, Pattern formation in twofrequency forced parametric waves, Phys. Rev. E 65, 036224 (2002).

[56] A. Kudrolli, B. Pier, and J. P. Gollub, Superlattice patterns in surface waves, Physica (Amsterdam) 123D, 99 (1998).

[57] Y. Ding and P. Umbanhowar, Enhanced Faraday pattern stability with three-frequency driving, Phys. Rev. E 73, 046305 (2006).

[58] A. M. Rucklidge, M. Silber, and A. C. Skeldon, Three-Wave Interactions and Spatiotemporal Chaos, Phys. Rev. Lett. 108, 074504 (2012).

[59] C. N. Likos, Effective interactions in soft condensed matter physics, Phys. Rep. 348, 267 (2001).

[60] A. J. Archer, M. J. Robbins, U. Thiele, and E. Knobloch, Solidification fronts in supercooled liquids: How rapid fronts can lead to disordered glassy solids, Phys. Rev. E 86, 031603 (2012).

[61] C. N. Likos, B. M. Mladek, D. Gottwald, and G. Kahl, Why do ultrasoft repulsive particles cluster and crystallize? Analytical results from density-functional theory, J. Chem. Phys. 126, 224502 (2007).

[62] R. Roth, Fundamental measure theory for hard-sphere mixtures: A review, J. Phys. Condens. Matter 22, 063102 (2010).

[63] A. C. Newell and Y. Pomeau, Turbulent crystals in macroscopic systems, J. Phys. A 26, L429 (1993).

[64] K. Jiang and W. Si, Stability of three-dimensional icosahedral quasicrystals in multi-component systems, arXiv: 1903.07859 . 